\title{
Numerical Simulation of the Effect of Heater Position on the Oxygen Concentration in the CZ Silicon Crystal Growth Process
}

\author{
Ying-Yang Teng, ${ }^{1}$ Jyh-Chen Chen,, ${ }^{2}$ Chung-Wei Lu, ${ }^{3}$ Cheng-Chuan Huang, ${ }^{2}$ Wan-Ting Wun, ${ }^{2}$ \\ Hsueh-I Chen, ${ }^{2}$ Chi-Yung Chen, ${ }^{4}$ and Wen-Chieh Lan $^{4}$ \\ ${ }^{1}$ Chung Shan Institute of Science and Technology (CSIST), No. 481, Sec. chia an, Zhongzheng Rd., Longtan, \\ Taoyuan County 32546, Taiwan \\ ${ }^{2}$ Department of Mechanical Engineering, National Central University, No. 300, Jhongda Rd., Jhongli City, \\ Taoyuan County 32001, Taiwan \\ ${ }^{3}$ Jen-Teh College, No. 79-9, Sha-Luen Hu, Hou-Loung, Miaoli County 35664, Taiwan \\ ${ }^{4}$ Sino-American Silicon Products Inc., No. 8. Industrial East Road 2, Hsinchu Science Park, Hsinchu County 30075, Taiwan
}

Correspondence should be addressed to Jyh-Chen Chen, jcchen@ncu.edu.tw

Received 28 August 2011; Accepted 23 December 2011

Academic Editor: C. W. Lan

Copyright ( 2012 Ying-Yang Teng et al. This is an open access article distributed under the Creative Commons Attribution License, which permits unrestricted use, distribution, and reproduction in any medium, provided the original work is properly cited.

\begin{abstract}
We perform numerical simulations to analyze the effect of the position of the heater on the thermal and flow fields and the oxygen concentration distribution during the industrial $\mathrm{Cz}$ silicon crystal growth process. The amount of oxygen released from the silica crucible to the silicon melt during the growth process can be lowered by adjusting the heater position to decrease the temperature on the crucible wall. During growth of the crystal body, there is a significant decrease in the gradient of the oxygen concentration along the melt-crystal interface due to the stronger Taylor-Proudman vortex, which is generated by the crucible and crystal rotation. There is a significant reduction in the average oxygen concentration at the melt-crystal interface for longer crystal lengths because of the lower wall temperature, smaller contact surface between the crucible wall and the melt and the stronger TaylorProudman vortex.
\end{abstract}

\section{Introduction}

The monocrystalline silicon (sc-Si) solar cell is one of the most important types of solar cells currently in use. However, to ensure grid parity, the quality of mono-Si wafers has to be improved even more. In mono-Si wafers, the oxygen impurity in combination with boron dopant induces light degradation of the solar cell. Therefore, to improve the efficiency of Si solar cells, the oxygen concentration in the crystal has to be controlled. The oxygen impurity originates mainly from the chemical reaction at the interface between the hot wall of the silica crucible and the Si melt. Part of the dissolved oxygen in the melt is incorporated into the crystal, while most oxygen evaporates on the free surface as $\mathrm{SiO}$, which is then transported away from the melt by the argon gas.

There have been many studies in the literature investigating how to control the oxygen concentration in a CZ system.
Machida and coworkers $[1,2]$ installed a gas controller and/ or a transverse magnetic field in their study of the effects of the argon gas flow rate and furnace pressure on the oxygen concentration in CZ grown silicon crystal. The oxygen concentration in the silicon crystal grown by a CZ system with a gas controller increases as the argon gas flow rate is reduced and the furnace pressure raised. When a transverse magnetic field is attached to a CZ furnace with a gas controller, there is a significant change in the flow pattern in the melt which causes an opposing trend of the dependence of the oxygen concentration in the CZ grown silicon crystal on the argon gas flow rate and the furnace pressure. Matsuo et al. [3] has shown by thermodynamic analysis that the oxygen concentration in the melt increases when the temperature of the silica wall increases. Chen et al. [4] have shown that the oxygen concentration in the CZ grown silicon crystal may be controlled by the rates of crucible and/or crystal rotation during 


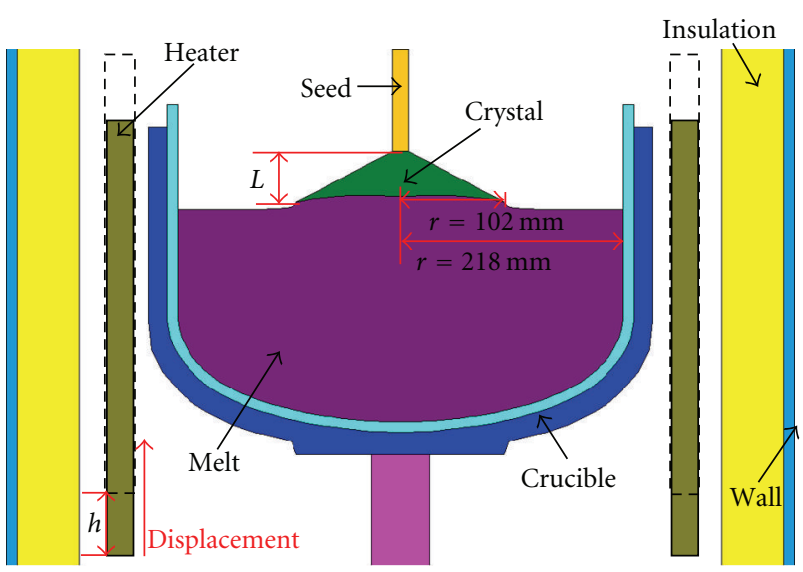

Figure 1: Schematic diagram of the major components of the CZ furnace, where $\mathrm{L}$ and $\mathrm{h}$ are the crystal length and the relative position of the heater in relation to the original position, respectively.

the growth. A lower oxygen concentration can be achieved in the crystal with a suitable combination of the crucible and crystal rotation rates.

The results of Matsuo et al. [3] show that the oxygen concentration level in the melt may be decreased by reducing the temperature of the silica crucible wall during the growth process. The crucible temperature is strongly affected by the arrangement of the heater in the CZ furnace. The appropriate positioning of the heater could possibly lower the temperature of the crucible wall. In the present study, a numerical investigation of the heat, flow fields, and oxygen concentration during the $\mathrm{CZ}$ silicon crystal growth process is carried out to determine the effect of the heater positioning on the oxygen concentration level in the crystal.

\section{Mathematical Model}

Figure 1 show a schematic representation of the conventional CZ furnace used in this study, where $h$ represents the relative position of the heater from the reference position. The furnace is assumed to be axis-symmetric and the growth process is assumed to be quasisteady. The silicon melt is considered to be a Newtonian fluid. The deformation of the free surface is ignored. The argon gas is assumed to be an ideal gas. The crystal diameter is selected to be 8 inches. The crucible, as shown in Figure 1, has a diameter of $438 \mathrm{~mm}$ and a height of $303 \mathrm{~mm}$. The amount of silicon in the crucible is $70.2 \mathrm{~kg}$ which is massive enough to grow a crystal $800 \mathrm{~mm}$ in length. The furnace pressure is kept at $2000 \mathrm{~Pa}$ with a $40 \mathrm{lpm}$ argon gas flowrate.

The differential equations governing heat and mass transports are as follows.

In the fluid:

$$
\begin{gathered}
\nabla \cdot\left(\rho_{i} \vec{u}_{i}\right)=0, \\
\left(\vec{u}_{i} \cdot \nabla\right) \rho_{i} \vec{u}_{i}=-\nabla p_{i}+\nabla \cdot \tau_{i}+\left(\rho_{i}-\rho_{i, 0}\right) \cdot \vec{g}, \\
C p_{i} \nabla \cdot\left(\rho_{i} \vec{u}_{i} T_{i}\right)=\nabla \cdot\left(k_{i} \nabla T_{i}\right), \\
\nabla \cdot\left(\rho_{i} \vec{u}_{i} C_{j}\right)=\nabla \cdot\left(D_{j} \nabla C_{j}\right),
\end{gathered}
$$

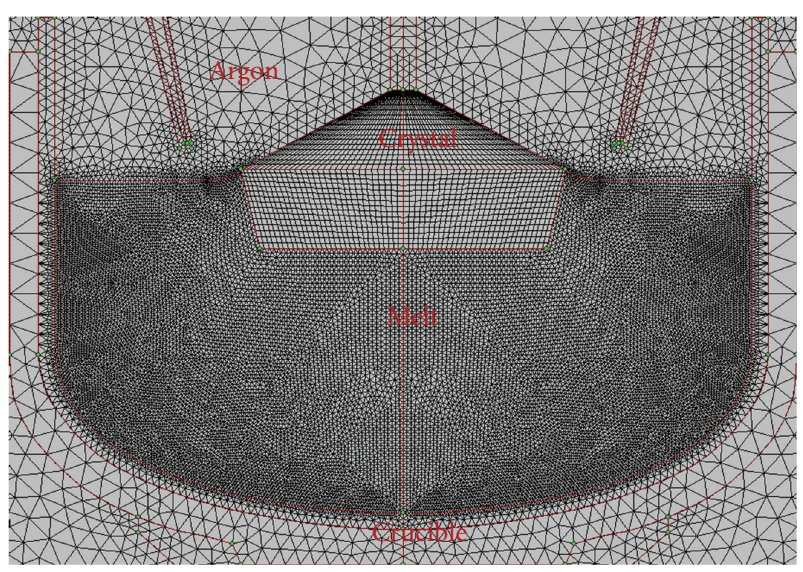

FIgURE 2: Distribution of the control volumes inside the furnace.

where $\rho, \rho_{0}, C p, \vec{u}, T, k, p, \tau, \vec{g}, C$, and $D$ are the density, reference density, heat capacity, velocity vector, temperature, thermal conductivity, pressure, stress tensor, gravitational acceleration, impurity concentration, and diffusion coefficient of the impurity, respectively. The subscript $i$ may be $g$ or 1 , where $g$ and 1 indicate the argon gas and silicon melt, respectively. The subscript $j$ is $\mathrm{O}$ or SiO, where $\mathrm{O}$ and $\mathrm{SiO}$ indicate the oxygen in the melt and silicon oxide in the argon, respectively.

In the heater:

$$
\nabla \cdot\left(k_{h} \nabla T_{h}\right)=\dot{q}
$$

where $\dot{q}$ is the heat generation from the heater.

The temperature at the melt/crystal interface is equal to the melting temperature of silicon, and the energy balance should satisfy the Stefan condition. The segregation effect of oxygen impurity is taken into account. The boundary conditions for the oxygen concentration at the crucible wall immersed into the silicon melt and for the oxygen concentration at free surface can be referred to our previous study [4]. The $\mathrm{SiO}$ concentration at the gas inlet is zero. The flux in the $\mathrm{SiO}$ concentration at the gas/solid interface is zero because the effect of deposition is ignored. The flow motion in the Si melt is in the transition regime [4]. The Reynolds average Navier-Stoke equation (RANS) is employed with the oneequation model [5] to simulate the turbulent motion inside the melt. To appropriately increase the dissipation rate near the wall, the Wolfshtein model [6] is used. To account for the turbulence suppression because of the crucible rotation effect, it is changed as a function of the mean strain and rotation rates [7]. For details of the boundary conditions and material properties, refer to $[4,8,9]$. The energy, continuity, momentum, and species equations with boundary conditions are solved with CGSim package, which is based on the finite volume method (FVM). Figure 2 shows the distribution of the control volumes inside the furnace. The effect of the control volume number is examined to find the best results providing sufficient accuracy and requiring less computational time for each case. The total number of the control volumes employed in the present computation is 28,587 . 


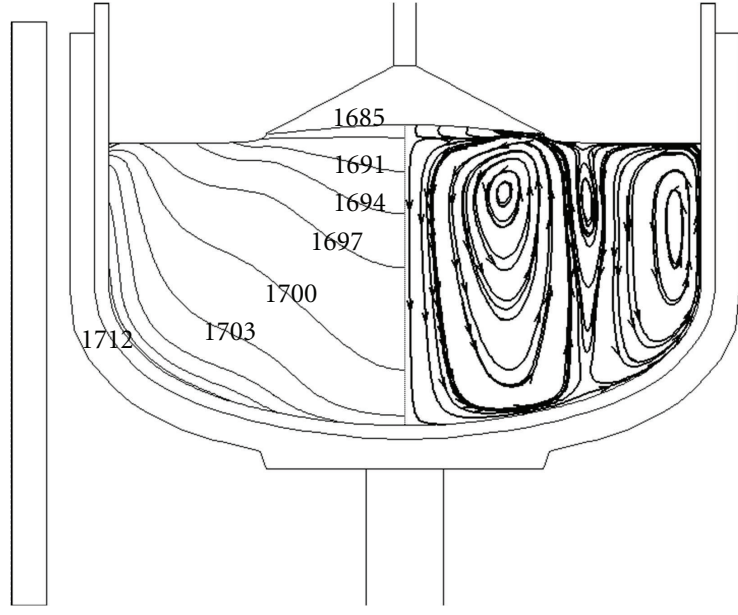

(a)

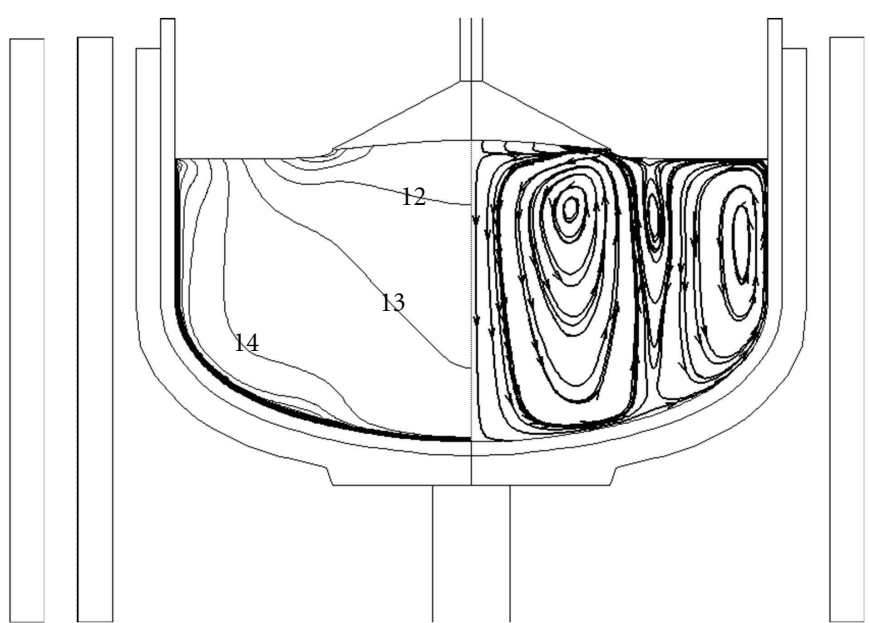

(b)

FIGURE 3: Distribution of the temperature, streamtrace, and oxygen concentration in the melt for $L=50 \mathrm{~mm}$ in a conventional CZ furnace $(h=0 \mathrm{~mm})$ : (a) isothermal lines (left) and streamtraces (right); (b) oxygen concentration isolines (left) and streamtraces (right). The units of temperature, velocity vector, and oxygen concentration are $\mathrm{K}, \mathrm{m} / \mathrm{s}$, and ppma, respectively. The spacing of the isothermal lines and concentration isolines are $3 \mathrm{~K}$ and 1 ppma, respectively.

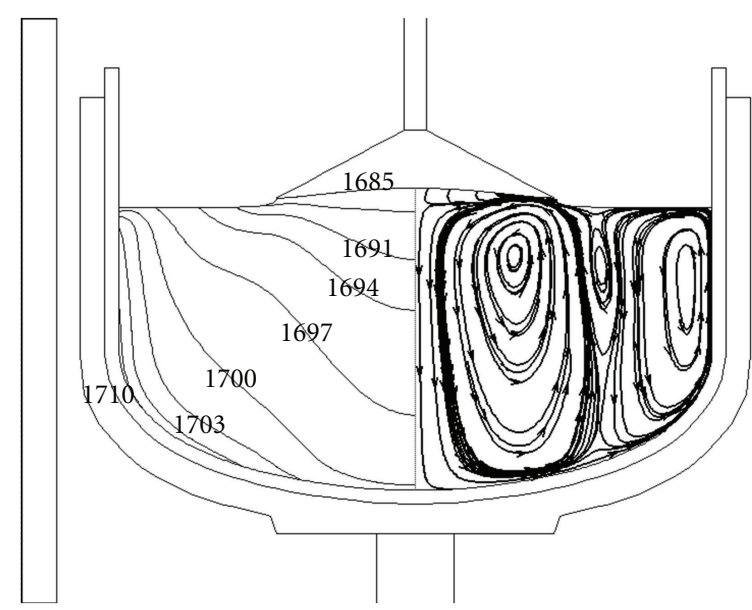

(a)
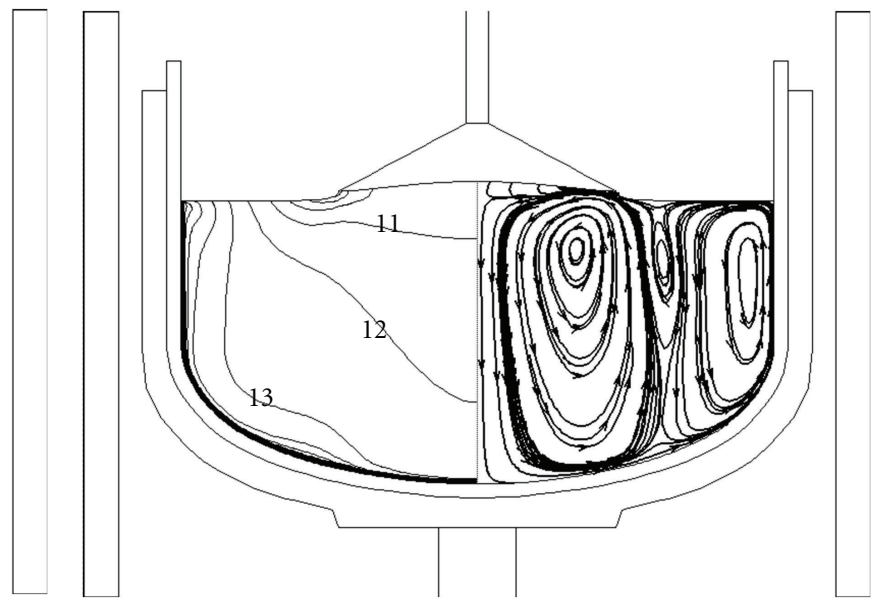

(b)

Figure 4: Distribution of temperature, streamtrace, and oxygen concentration in the melt for $L=50 \mathrm{~mm}$ in a conventional CZ furnace $(h=50 \mathrm{~mm})$ : (a) isothermal lines (left) and streamtraces (right); (b) oxygen concentration isolines (left) and stream traces (right). The units of temperature, velocity vector, and oxygen concentration are $\mathrm{K}, \mathrm{m} / \mathrm{s}$, and ppma, respectively. The spacing of the isothermal lines and concentration isolines are $3 \mathrm{~K}$ and 1 ppma, respectively.

\section{Results and Discussion}

According to the study of Chen et al. [4], there is an optimum combination of crucible and crystal rotations for obtaining the lowest oxygen concentration for a CZ furnace. In this study, the crystal and crucible rotation rates are fixed at 13 and $-3 \mathrm{rpm}$, respectively, which indicate the best operating conditions for the lowest oxygen concentration obtained by Chen et al. [4]. The maximum distance of upward movement of the heater from $h=0 \mathrm{~mm}$ without contact to the side insulation for the present furnace is $50 \mathrm{~mm}$. Three different heater positions of $h=0,30$, and $50 \mathrm{~mm}$ are considered here.
During the crystal growth, the heater position is fixed and the crucible is moved upwards to keep the predetermined gap between the free surface of the silicon melt and the heat shield. The numerical computations are carried out for different crystal lengths $L$.

Figures 3 and 4 show the temperature, streamtrace, and oxygen concentration distributions in the melt for $L=$ $50 \mathrm{~mm}$ (crystal crown was formed) with $h=0$ and $50 \mathrm{~mm}$. There are 3 vortices in the melt: the buoyancy-thermocapillary vortex near the wall, Taylor-Proudman vortex under the crystal, and secondary vortex between them. The oxygen impurity originating from the silica crucible wall is diffused 


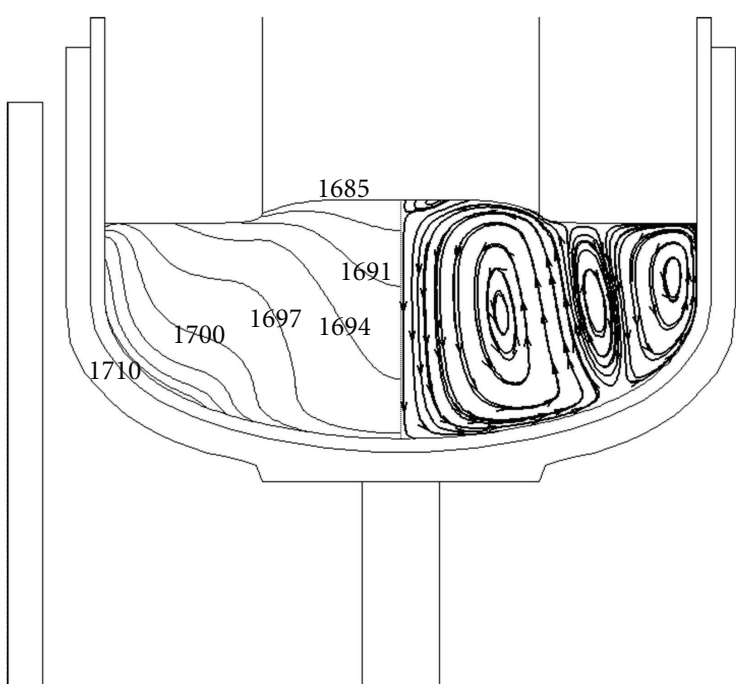

(a)

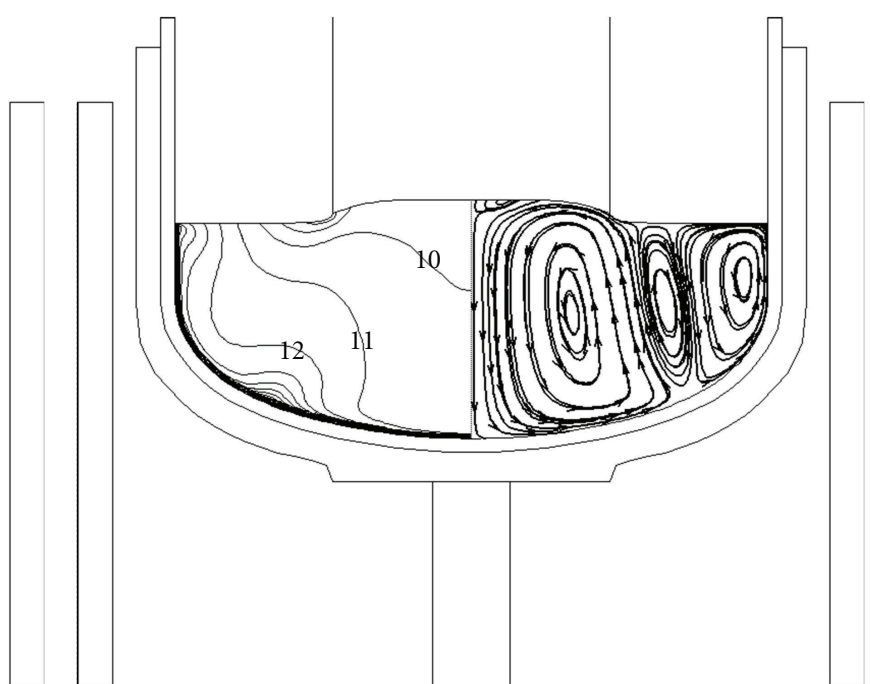

(b)

Figure 5: Distribution of temperature, streamtrace, and oxygen concentration in the melt for $L=300 \mathrm{~mm}$ in a conventional CZ furnace ( $h=0 \mathrm{~mm}$ ): (a) isothermal lines (left) and streamtraces (right); (b) oxygen concentration isolines (left) and streamtraces (right). The units of temperature, velocity vector, and oxygen concentration are $\mathrm{K}, \mathrm{m} / \mathrm{s}$, and ppma, respectively. The spacing of the isothermal lines and concentration isolines are $3 \mathrm{~K}$ and 1 ppma, respectively.

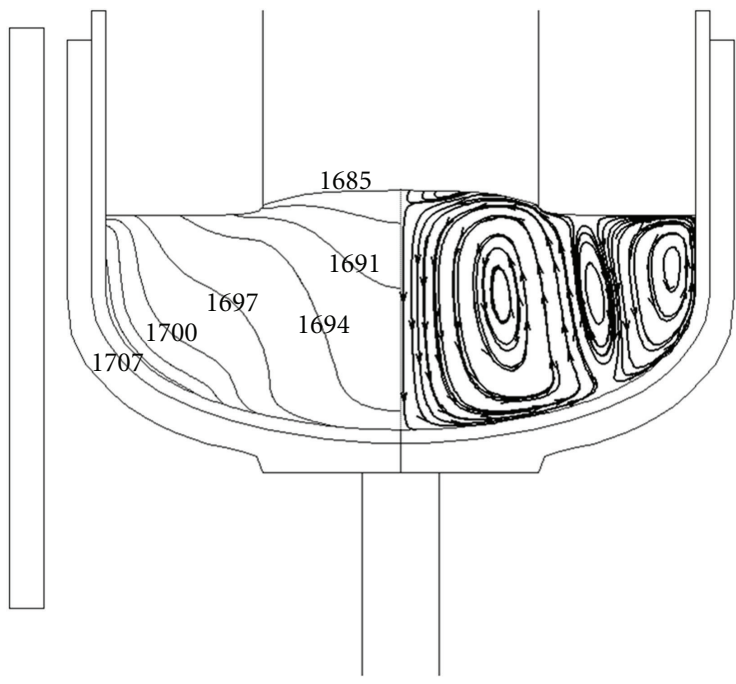

(a)

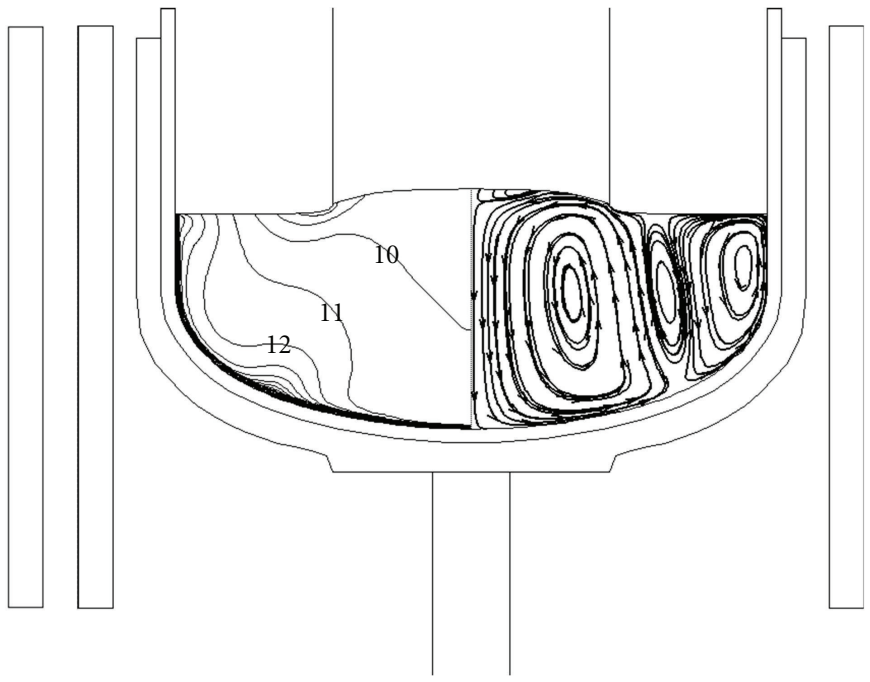

(b)

FIgURE 6: Distribution of temperature, streamtrace, and oxygen concentration in the melt for $L=300 \mathrm{~mm}$ in a conventional CZ furnace $(h=50 \mathrm{~mm})$ : (a) isothermal lines (left) and streamtraces (right); (b) oxygen concentration isolines (left) and streamtraces (right). The units of temperature, velocity vector, and oxygen concentration are $\mathrm{K}, \mathrm{m} / \mathrm{s}$, and ppma, respectively. The spacing of the isothermal lines and concentration isolines are $3 \mathrm{~K}$ and 1 ppma, respectively.

into the silicon melt. This is prevented at the crystal-melt interface by the suppression of the flow motion of the TaylorProudman vortex and carried to the free surface of the melt by the buoyancy-thermocapillary vortex. The oxygen impurity at the free surface can vaporize to form $\mathrm{SiO}$ gas which is removed from the furnace by the motion of the argon gas. The maximum temperature $T_{\max }$ at the crucible wall appears at the intersection of the vertical and bottom wall. For $h=0 \mathrm{~mm}, T_{\max }$ is $1712 \mathrm{~K}$, which is higher than that for $h=50 \mathrm{~mm}\left(T_{\max }=1710 \mathrm{~K}\right)$. The melt flow patterns are similar due to the small difference in the maximum temperature between $h=0$ and $50 \mathrm{~mm}$ and the same combination of rotation rates of crucible and crystal. Therefore, the distributions of the isotherms and oxygen concentration isolines in the melt are also similar. The amount of oxygen impurity released from the crucible wall decreases as the temperature of the crucible wall and the amount of contact surface of the melt and the crucible wall decrease. Since 


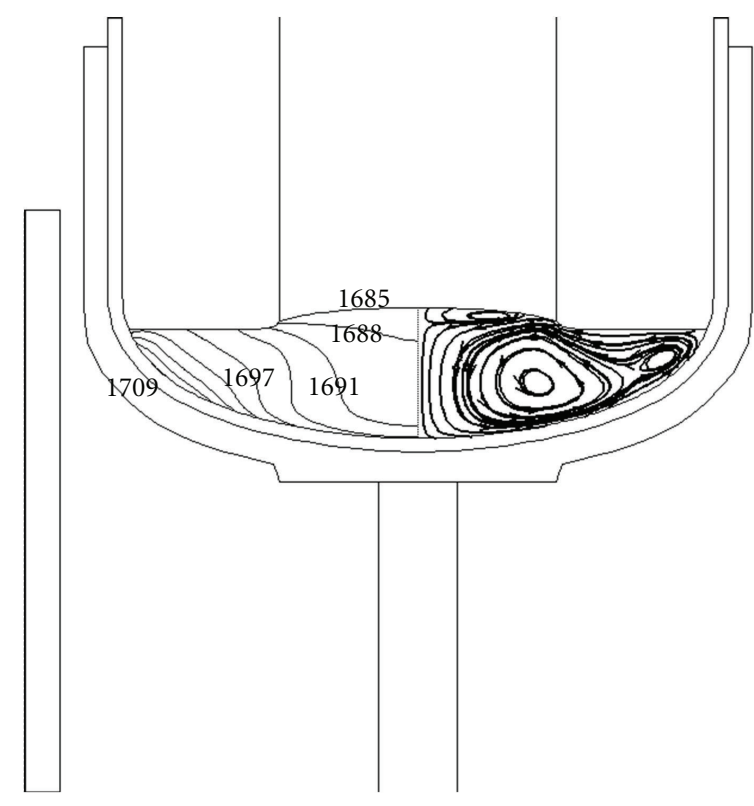

(a)

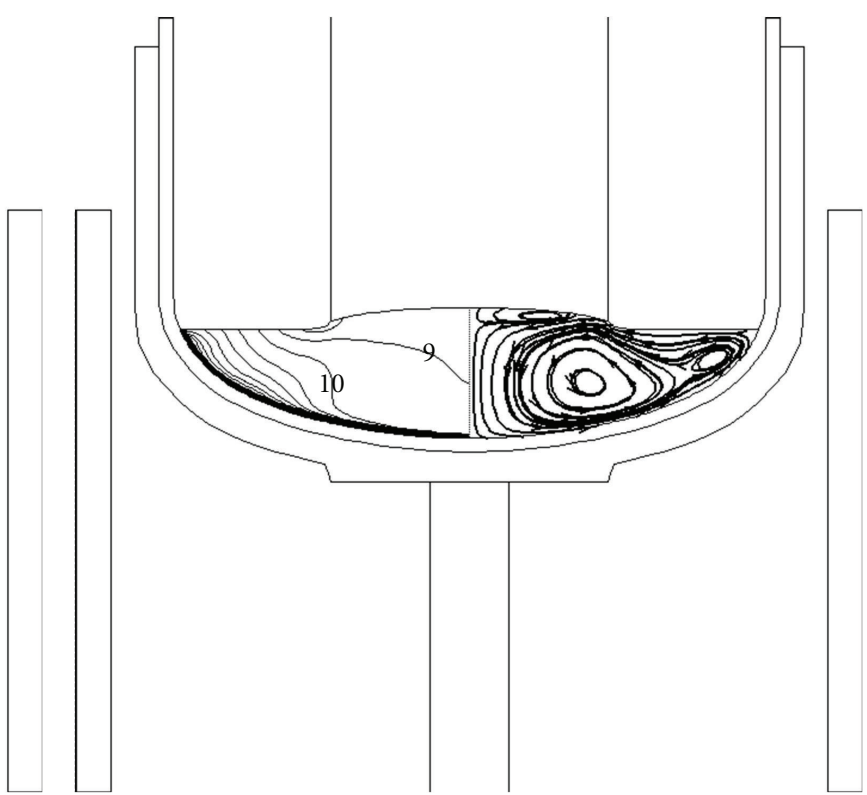

(b)

FIgURE 7: Distribution of temperature, streamtrace, and oxygen concentration in the melt for $L=700 \mathrm{~mm}$ in a conventional CZ furnace ( $h=0 \mathrm{~mm}$ ): (a) isothermal lines (left) and streamtraces (right); (b) oxygen concentration isolines (left) and streamtraces (right). The units of temperature, velocity vector, and oxygen concentration are $\mathrm{K}, \mathrm{m} / \mathrm{s}$, and ppma, respectively. The spacing of the isothermal lines and concentration isolines are $3 \mathrm{~K}$ and 1 ppma, respectively.

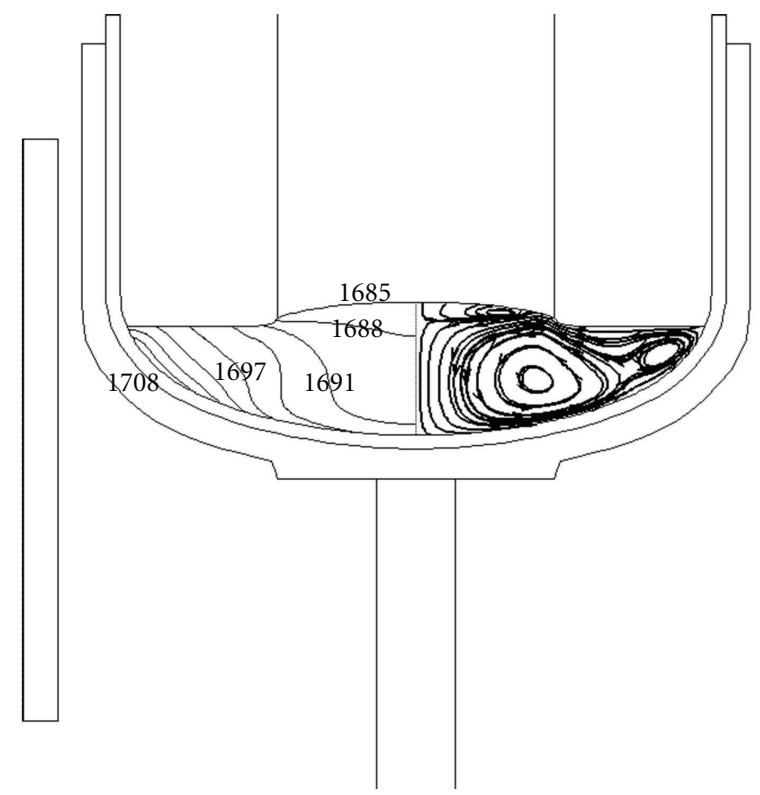

(a)

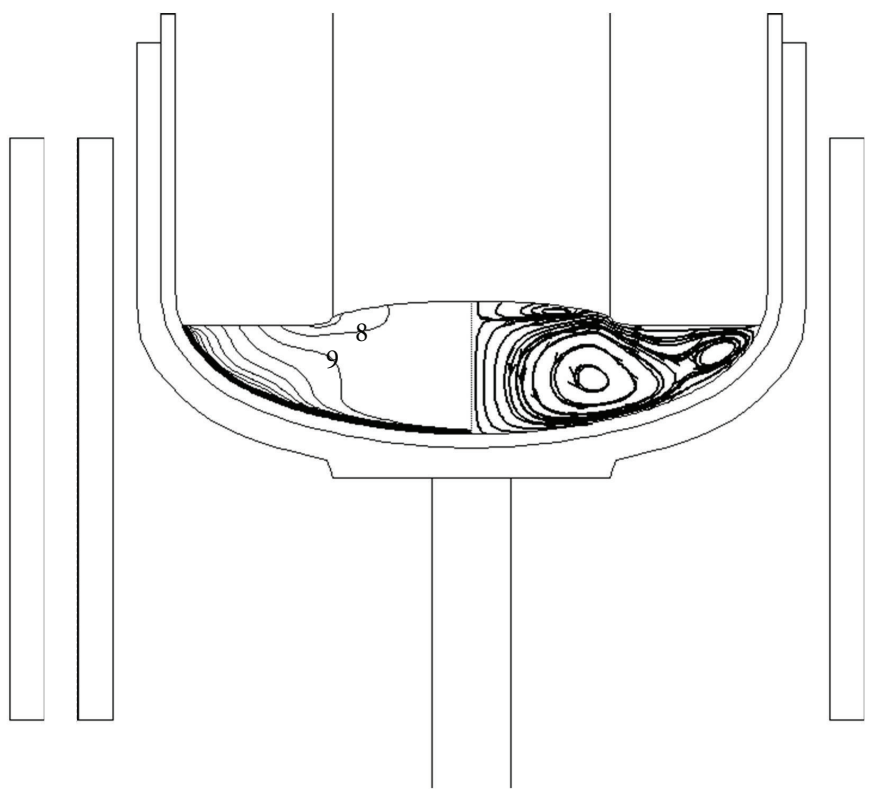

(b)

FIGURE 8: Distribution of temperature, streamtrace, and oxygen concentration in the melt for $L=700 \mathrm{~mm}$ in a conventional CZ furnace $(h=50 \mathrm{~mm})$ : (a) isothermal lines (left) and streamtraces (right); (b) oxygen concentration isolines (left) and streamtraces (right). The units of temperature, velocity vector, and oxygen concentration are $\mathrm{K}, \mathrm{m} / \mathrm{s}$, and ppma, respectively. The spacing of the isothermal lines and concentration isoline are $3 \mathrm{~K}$ and 1 ppma, respectively.

the maximum temperature of $h=50 \mathrm{~mm}$ is lower, the magnitude of oxygen concentration is smaller.

It can be seen in Figures 5 and 6 that the flow, temperature, and oxygen concentration patterns for $L=300 \mathrm{~mm}$ are similar to those for $h=0$ and $50 \mathrm{~mm}$. Since the depth of the melt for $L=300 \mathrm{~mm}$ is much smaller than when $L=$ $50 \mathrm{~mm}$, there is a significant reduction in the strength of the buoyancy-thermocapillary vortex for $L=300 \mathrm{~mm}$ in comparison to the case for $L=50 \mathrm{~mm}$. Hence, the TaylorProudman vortex for the $L=300 \mathrm{~mm}$ case becomes stronger. 


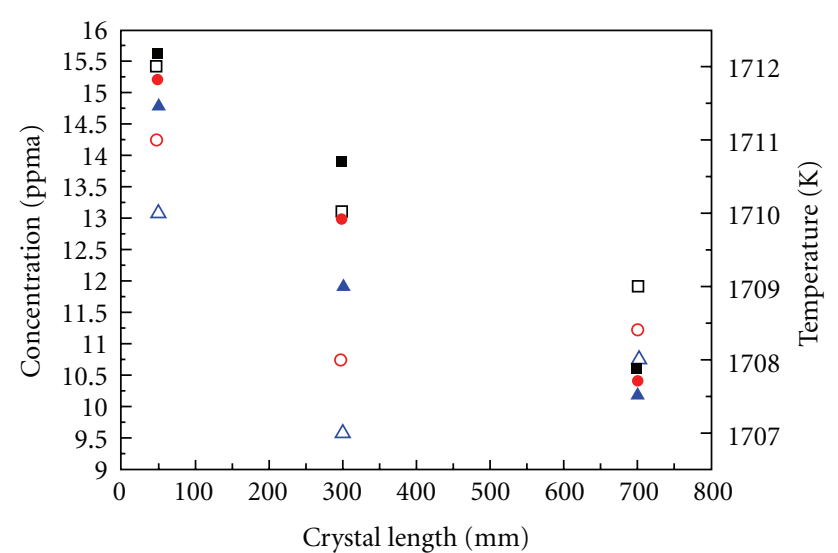

$\begin{array}{ll}\square h=0 \mathrm{~mm} & \square h=0 \mathrm{~mm} \\ \bullet \quad h=30 \mathrm{~mm} & \bigcirc h=30 \mathrm{~mm} \\ \triangle h=50 \mathrm{~mm} & \triangle h=50 \mathrm{~mm}\end{array}$

Figure 9: Average oxygen concentration at the crystal-melt interface and maximum temperature of crucible wall versus crystal length $L$.

The effect of the Taylor-Proudman vortex is totally dominant for $L=700 \mathrm{~mm}$ (Figures 7 and 8). The temperature and oxygen gradients near the crystal-melt interface become smaller when the length of crystal increases. On the other hand, the magnitude of the temperature and oxygen concentration in the melt decreases for longer crystal lengths (Figures 3-8).

The average oxygen concentration at the crystal-melt interface is defined as the total oxygen concentration at the crystal-melt interface, which is obtained by integrating the oxygen concentration over the entire crystal-melt interface, divided by the crystal-melt area. Figure 9 displays the variation of the average oxygen concentration at the crystal-melt interface and the maximum wall temperature with the crystal height for $h=0,30$, and $50 \mathrm{~mm}$. For a fixed crystal length, the average oxygen concentration decreases as $h$ increases. It can be seen that the difference of the maximum temperature for $h=0,30$, and $50 \mathrm{~mm}$ is more significant for $L=300 \mathrm{~mm}$. This may explain the difference in average oxygen concentration for $L=300 \mathrm{~mm}$ (Figure 9). On the other hand, the heater power increases as the heater position moves upward. The reduction in the temperature of the crucible wall, the contact surface of the melt and the crucible wall and the oxygen gradients along the melt-crystal interface indicates a reduction in the average oxygen concentration when the crystal length increases. As the crystal length increases from $L=50 \mathrm{~mm}$ to $L=300 \mathrm{~mm}$, the input power decreases. When the crystal length further increases from $L=300 \mathrm{~mm}$ to $L=$ $500 \mathrm{~mm}$, it increases.

\section{Conclusion}

The heater position is one of the important factors affecting the temperature at the crucible wall. In the present study, the oxygen concentration distributions for different heater positions are simulated. When the crucible and crystal rotation rates are determined, the flow pattern in the melt is similar for different heater positions. The oxygen concentration in the melt will be dependent on the temperature of the crucible wall and the amount of contact between the surface of the crucible wall and the melt. For cases considered in the present study, when the heater moves upwards from the reference position, the oxygen concentration becomes lower due to the lower crucible wall temperature. For larger crystal lengths, there is a notable reduction in the length of the gradients of oxygen concentration along the melt-crystal interface, since the Taylor-Proudman vortex generated by the crucible and crystal rotation is more dominant, while the wall temperature and the contact surface of the crucible wall and the melt become lower. Therefore, the oxygen concentration in the melt decreases significantly as the crystal grows longer.

\section{Acknowledgment}

The authors would like to thank the National Science Council of Taiwan, for the support of this work through Grant No. NSC-98-2622-8-008-002-A1.

\section{References}

[1] N. Machida, Y. Suzuki, K. Abe, N. Ono, M. Kida, and Y. Shimizu, "The effects of argon gas flow rate and furnace pressure on oxygen concentration in Czochralski-grown silicon crystals," Journal of Crystal Growth, vol. 186, no. 3, pp. 362-368, 1998.

[2] N. Machida, K. Hoshikawa, and Y. Shimizu, "Effects of argon gas flow rate and furnace pressure on oxygen concentration in Czochralski silicon single crystals grown in a transverse magnetic field," Journal of Crystal Growth, vol. 210, no. 4, pp. 532-540, 2000.

[3] H. Matsuo, R. Bairava Ganesh, S. Nakano et al., "Thermodynamical analysis of oxygen incorporation from a quartz crucible during solidification of multicrystalline silicon for solar cell," Journal of Crystal Growth, vol. 310, no. 22, pp. 4666-4671, 2008.

[4] J. C. Chen, Y. Y. Teng, W. T. Wun et al., "Numerical simulation of oxygen transport during the $\mathrm{CZ}$ silicon crystal growth process," Journal of Crystal Growth, vol. 318, no. 1, pp. 318-323, 2011.

[5] N. G. Ivanov, A. B. Korsakov, E. M. Smirnov et al., "Analysis of magnetic field effect on 3D melt flow in CZ Si growth," Journal of Crystal Growth, vol. 250, no. 1-2, pp. 183-188, 2003.

[6] M. Wolfshtein, "The velocity and temperature distribution in one-dimensional flow with turbulence augmentation and pressure gradient," International Journal of Heat and Mass Transfer, vol. 12, no. 3, pp. 301-318, 1969.

[7] "CGSim Flow Module," Ver. 3.11.1, Theory Manual, STR, Inc., Richmond, Va, USA, 2010, http://www.str-soft.com/.

[8] Y. Y. Teng, J. C. Chen, C. W. Lu, and C. Y. Chen, "The carbon distribution in multicrystalline silicon ingots grown using the directional solidification process," Journal of Crystal Growth, vol. 312, no. 8, pp. 1282-1290, 2010.

[9] Y. Y. Teng, J. C. Chen, C. W. Lu, H. I. Chen, C. Hsu, and C. Y. Chen, "Effects of the furnace pressure on oxygen and silicon oxide distributions during the growth of multicrystalline silicon ingots by the directional solidification process," Journal of Crystal Growth, vol. 318, p. 224, 2011. 


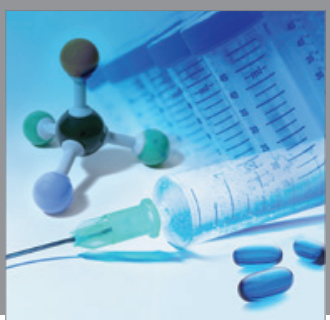

International Journal of

Medicinal Chemistry

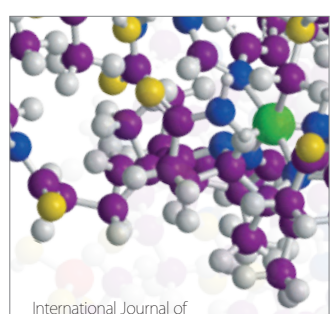

Carbohydrate Chemistry

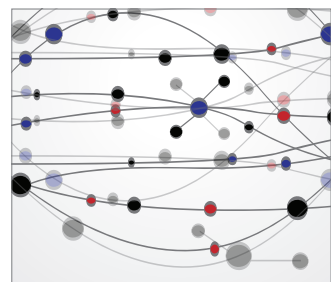

The Scientific World Journal
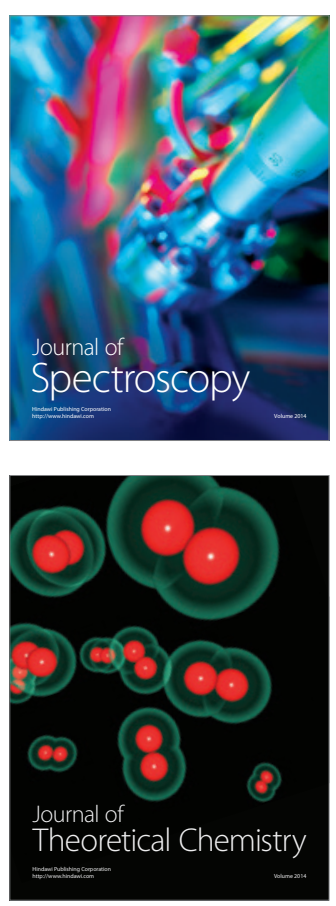
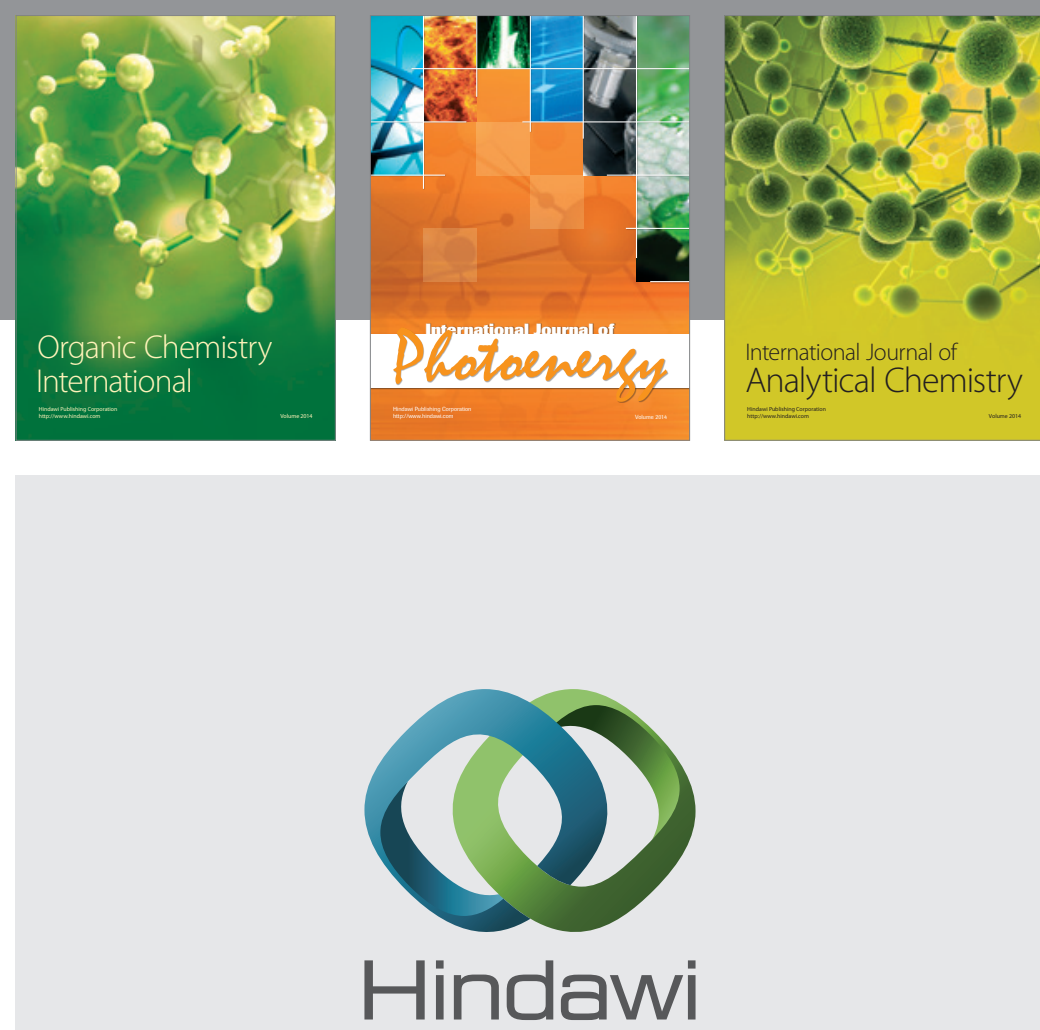

Submit your manuscripts at

http://www.hindawi.com
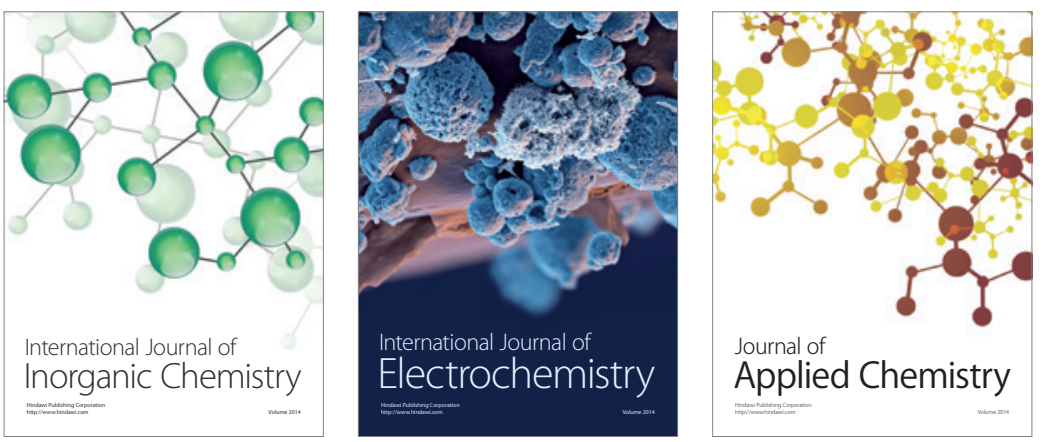

Journal of

Applied Chemistry
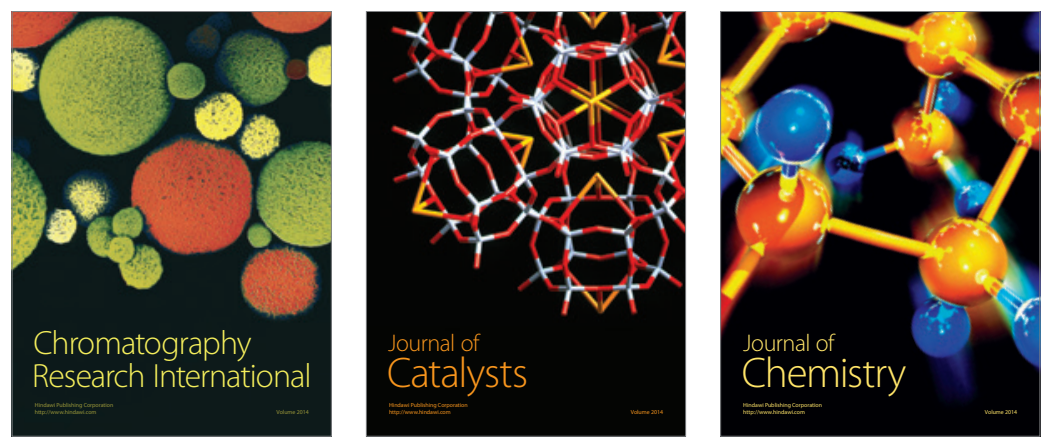
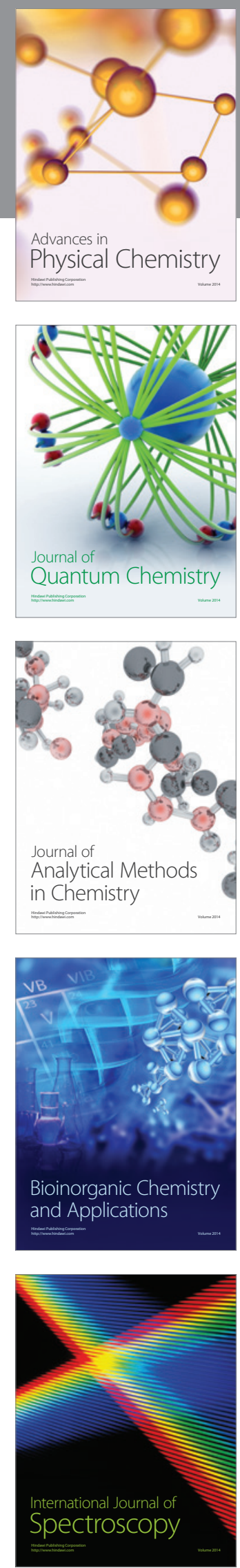